\title{
Image Classification with Indicator Kriging Error Comparison
}

\author{
Tuan D. Pham \\ Aizu Research Cluster for Medical Engineering and Informatics \\ Research Center for Advanced Information Science and Technology \\ The University of Aizu \\ Aizuwakamatsu, Fukushima, 965-8580, Japan \\ tdpham@u-aizu.ac.jp
}

\begin{abstract}
Methods for classification of images are of an important research area in image processing and pattern recognition. In particular, image classification is playing an increasingly important role in medicine and biology with respect to medical diagnoses and drug discovery, respectively. This paper presents a new method for image classification based on the frameworks of fuzzy sets and geostatistics. The proposed method was applied to the automated detection of regions of mitochondria in microscope images. The high correction rate of detecting the locations of the mitochondria in a complex environment obtained from the proposed method suggests its effectiveness and its better performance than several other existing algorithms.
\end{abstract}

Keywords: Image classification; Fuzzy sets; Indicator kriging; Uncertainty.

\section{Introduction}

Image classification is an important area of research in pattern recognition. It is an act of receiving some unknown raw image data and making a decision to assign the raw image to one of the known image patterns. Techniques for image classification have been widely developed to provide solutions to different domain problems. In general, after a process of extracting features from an image, the classifcation can be carried out by either a decision-theoretic approach, including template matching, optimal statistical classifiers, and machine-learning; or structural methods, including shape matching, string matching, and syntactic recognition [1]. In particular, detection and classification of molecular and medical images has been a long-pursued research in the disciplinary field of engineering and computer science in life sciences [23. However, there is always a strong demand for exploring appropriate feature extraction methods for the automated identification of particular types of objects or regions of interest in cell biology and medicine with different levels of technical challenge, ranging from the detection of cells nuclei [4] to subcellular patterns [5]. If different types of the images can be automatically distinguished by computerized methods, such

A. Elmoataz et al. (Eds.): ICISP 2014, LNCS 8509, pp. 433-440, 2014.

(C) Springer International Publishing Switzerland 2014 
an ability can help researchers to quickly and accurately study cell function to discover mechanisms underlying complex diseases, and carry out spatial modeling and simulation of biological signaling pathways, which may identify critical organelles attributing to the regulation of the cellular process within the intracellular space. Popular methods which have been applied for characterizing molecular and medical images are known as image feature extraction techniques. Texture is among the most useful features for molecular image analysis because in many cases such images of normal and diseased stages have strong similarity in or exhibit the absence of size and shape which can be a challenge for geometryinvariant analysis [67]. Here we are therefore interested in developing a new image feature analysis by means of spatial uncertainty modeling using geostatistics and fuzzy sets for the automated detection of mitochondria in FIB-SEM images.

The rest of this paper is organized as follows. Section 2 presents the proposed approach for quantifying spatial uncertainty in the intracellular space, which can be used for template-matching based clasification. Experiments on the performance of different feature analysis methods are discussed in Section 3. Finally, Section 4 is the conclusion of the finding.

\section{Methods}

The purpose of the proposed approach is to model and quantify integrated uncertainty inherently existing in images, which is subject to both imprecision and spatial random processes. Such an uncertainty measure can then be used in terms of error comparison for image classification. The derivation of the approach is described as follows. The intensity distibution of pixels in an image is considered as a spatial random process, which can be modeled with the indicator kriging formalism of geostatistics. The indicator kriging has been applied as a natural tool for determining a non-parametric conditional probability distribution of categorical data [8]. An interest in this study is to utilize indicator kriging to construct local spatial distributions of uncertainty in an image. Let $f_{i}$ be the intensity value of a pixel located at $i, i=1, \ldots, L \times W, L \times W$ is the size of the image. Here, the purpose of applying the indicator formalism is to estimate the probability distribution of uncertainty at unsampled location $i$. The cummulative distribution function is usually estimated with a set of cut-off thresholds $z_{t}, t=1, \ldots, T$; and the probabilities are then determined by coding the data as binary indicator values. The indicator coding at location $i$ is defined as follows [8]

$$
I(i, T)=P\left(f_{i} \leq z_{t}\right)=\left\{\begin{array}{lll}
1 & : & f_{i} \leq z_{t} \\
0 & : & \text { otherwise. }
\end{array}\right.
$$

Using thresholds $z_{t}, t=1, \ldots, T$, as values in the range of the image intensity does not conveniently offer a procedure for modeling spatial uncertainty in an image. Therefore, instead of using $z_{t}$, the fuzzy $c$-means algorithm [9] is applied to arbitrarily produce image partitions or clusters $\mathbf{v}_{j}, j=1, \ldots, c$, which allows 
every pixel to belong to every partition with different fuzzy membership grades, and apply a series of $\alpha$-level cuts, $\alpha \in[0,1]$, to code the categorical pixels as follows:

$$
I_{j}(i, \alpha)=\left\{\begin{array}{lll}
1 & : & \mu_{i j} \geq \alpha \\
0 & : & \text { otherwise }
\end{array}\right.
$$

where $I_{j}(i ; \alpha)$ is the indicator that codes the assignment of $f_{i}$ to cluster $\mathbf{v}_{j}$ having a fuzzy membership grade being equal to or greater than $\alpha$.

The next step of the indicator kriging formalism is the determination of the cumulative distribution function (CDF), which characterizes the probability of $f_{i}$ belonging to $\mathbf{v}_{j}$ with a membership value of being greater or equal to $\alpha$, and can be mathematically expressed as

$$
F_{j}(i, \alpha)=P\left(\mu_{i j} \geq \alpha\right)
$$

Taking advantage of the available information of $M$-neighboring data, the conditional CDF is

$$
F_{j}[(i, \alpha) \mid M]=P\left[\left(\mu_{i j} \geq \alpha\right) \mid M\right]
$$

where $M$ is the number of neighboring pixels of $i$.

The CDF according to the indicator expressed in Eq. (2) can be estimated using the ordinary kriging [10, and the result of indicator kriging is a model of spatial uncertainty at the pixel at location $i$ :

$$
\hat{F}_{j}[(i, \alpha) \mid M]=\sum_{m=1}^{M} w_{m}(i, \alpha) I_{j}(i, \alpha)
$$

where $w_{m}(i, \alpha)$ is the ordinary kriging weight that indicates the influence of neighboring pixel $m$ over pixel $i$ with respect to level cut $\alpha$. These weights can be optimally determined by the ordinary kriging system of equations [1011]:

$$
\left\{\begin{array}{l}
\sum_{m=1}^{M} w_{m}(i, \alpha) \gamma_{\alpha, m, m^{\prime}}+\lambda=\gamma_{\alpha, m, i}, m^{\prime}=1, \ldots, M \\
\sum_{m=1}^{M} w_{m}(i, \alpha)=1,
\end{array}\right.
$$

where $\lambda$ is a Lagrange multiplier, $\gamma_{\alpha, m, m^{\prime}}$, with a lag of absolute difference $\mid m-$ $m^{\prime} \mid=h$, is the semivariogam of the indicator $I_{j}(i, \alpha)$ expressed in Eq. (2), and is defined as the expected value [8]:

$$
\gamma_{\alpha}(h)=\frac{1}{2} E\left\{\left[I_{j}(m, \alpha)-I_{j}(m+h, \alpha)\right]^{2}\right\}
$$

The indicator semivariogram that is experimentally calculated for lag distance $h$ is defined as the average squared difference of values separated by $h$ :

$$
\hat{\gamma}_{\alpha}(h)=\frac{1}{2 N(h)} \sum_{N(h)}\left[I_{j}(m, \alpha)-I_{j}(m+h, \alpha)\right]^{2},
$$

where $N(h)$ is the number of pairs for lag $h$. 
An implicit assumption of the ordinary kriging system having presented is that the underlying statistics is invariant in space under translation. Such a property is known as statistically stationary. However, statistical stationarity is a property of a random function but not an inherent property of real data [12. This nonstationary property is also true for biomedical images in which biological elements can have different variations of the image intensity and the mean of the image changes locally. Here, kriging with a nonstationary mean is applied to enhance the reliability of the estimate of the kriging weights. This technique is called universal kriging (UK) [11,13.

In ordinary kriging, the estimation is carried out with the error variable from a stationary mean that must be known at all positions and can be set as the global mean or modeled with a drift or a local trend. A local mean with a drift $m(u)$ can be modeled as a linear combination of the geometrical coordinates of the pixels with a local neighborhood as

$$
m(u)=\sum_{k=0}^{K} a_{k} L_{k}(u),
$$

where $a_{k}$ are unknown drift coefficients, $L_{0}(u)=1$ (constant function for the constant-mean case), and $L_{k}(u), k=1, \ldots, K$, are the polynomials or basis functions, which can be modeled as the first-degree or second-degree terms as follows, respectively.

$$
\begin{gathered}
m(u)=a_{0}+\sum_{k=1}^{K}\left(a_{1} x_{1 k}+a_{2} x_{2 k}\right) \\
m(u)=a_{0}+\sum_{k=1}^{K}\left(a_{1} x_{1 k}+a_{2} x_{2 k}+a_{3} x_{1 k}^{2}+a_{4} x_{2 k}^{2}+a_{5} x_{1 k} x_{2 k}\right)
\end{gathered}
$$

where $x_{1 k}$ and $x_{2 k}$ are the pixel coordinates in row-wise and column-wise of $f_{k}$, respectively.

The drift effect can be incorporated into the ordinary kriging system to find kriging weights as additional constraints. Solving this extended set of simultaneous equations, a set of universal kriging weights that model the drift within the local neighbors around the location of the unknown value. In general, the UK system can be expressed with the following matrix structure

$$
\left[\begin{array}{ll}
\mathbf{G}^{*} & \mathbf{L} \\
\mathbf{L}^{T} & \mathbf{0}
\end{array}\right]\left[\begin{array}{l}
\mathbf{w}^{*} \\
\mathbf{m}
\end{array}\right]=\left[\begin{array}{c}
\mathbf{g}^{*} \\
\mathbf{l}
\end{array}\right]
$$

where $\mathbf{G}^{*}, \mathbf{w}^{*}$, and $\mathbf{g}^{*}$ are the $\mathbf{G}$ without the last row and last column, $\mathbf{w}$ without the last row, and $\mathbf{g}$ without the last row as defined for the ordinary kriging system, respectively; 


$$
\mathbf{L}=\left[\begin{array}{cccc}
1 & L_{11} & \cdots & L_{K 1} \\
1 & L_{12} & \cdots & L_{K 2} \\
\cdot & \cdot & \cdots & \cdot \\
\cdot & \cdot & \cdots & \cdot \\
\cdot & \cdot & \cdots & \cdot \\
1 & L_{1 n} & \cdots & L_{K n}
\end{array}\right]
$$

where $L_{11}$ denotes $L_{1}\left(f_{1}\right)$,

$$
\mathbf{m}=\left[\beta_{0} \beta_{1} \cdots \beta_{K}\right]^{T}
$$

where $\beta_{k}, k=0, \ldots, K$ are the $K+1$ additional Lagrange multipliers, and

$$
\mathbf{l}=\left[\begin{array}{llll}
1 & L_{1 u} & \cdots & L_{K u}
\end{array}\right]^{T} .
$$

Let $\mathbf{A}=\left[\begin{array}{ll}\mathbf{G}^{*} & \mathbf{L} \\ \mathbf{L}^{T} & \mathbf{0}\end{array}\right], \mathbf{w}=\left[\begin{array}{c}\mathbf{w}^{*} \\ \mathbf{m}\end{array}\right]$, and $\mathbf{b}=\left[\begin{array}{c}\mathbf{g}^{*} \\ \mathbf{l}\end{array}\right]$. The error variance of the indicator kriging estimation is

$$
\sigma^{2}=\mathbf{w}^{T} \mathbf{b}
$$

A comparison between the two variance errors of two images, $\mathcal{I}$ and $\mathcal{I}^{\prime}$, can be defined as

$$
D\left(\mathbf{w}, \mathbf{w}^{\prime}\right)=\left(\mathbf{w}^{\prime}\right)^{T} \mathbf{b}-\mathbf{w}^{T} \mathbf{b}
$$

where $\mathbf{w}$ and $\mathbf{b}$ are computed from image $\mathcal{I},\left(\mathbf{w}^{\prime}\right)$ is from image $\mathcal{I}^{\prime}$. Since $\mathbf{w}^{T} \mathbf{b}$ is the minimized kriging error variance obtained for $\mathcal{I}$, thus $\left(\mathbf{w}^{\prime}\right)^{T} \mathbf{b} \geq \mathbf{w}^{T} \mathbf{b}$. For a perfect match between $\mathcal{I}$ and $\mathcal{I}^{\prime}$, the errors are identical and Eq. (14) results in a zero difference. The error difference gets larger for a larger mismatch between $\mathcal{I}$ and $\mathcal{I}^{\prime}$.

The discussion completes with a decision procedure for a computed set of the kriging error variances for $\omega_{s}, s=1, \ldots, K$, classes as follows. Let fuzzy cluster centers $\mathbf{v}_{1}>\ldots>\mathbf{v}_{c}$, and $\alpha$-level cuts $\alpha_{1}>\ldots>\alpha_{b}$ (if such orders do not exist, then the orders are rearranged). Also let $\sigma_{s}^{2}\left(\mathcal{I}, \alpha_{r}, \mathbf{v}_{j}\right), r=1, \ldots b, j=1, \ldots, c$, be the set of kriging error variances of image $\mathcal{I}$ obtained by using $\alpha$-level cut $\alpha_{r}$ and fuzzy partition $\mathbf{v}_{j}$ for class $\omega_{s}$ :

$$
\begin{aligned}
& L_{1}\left(\omega_{s}\right)=\left[\sigma_{s}^{2}\left(\mathcal{I}, \alpha_{1}, \mathbf{v}_{1}\right), \ldots, \sigma_{s}^{2}\left(\mathcal{I}, \alpha_{r}, \mathbf{v}_{1}\right)\right], \ldots, \\
& L_{c}\left(\omega_{s}\right)=\left[\sigma_{s}^{2}\left(\mathcal{I}, \alpha_{1}, \mathbf{v}_{c}\right), \ldots, \sigma_{s}^{2}\left(\mathcal{I}, \alpha_{r}, \mathbf{v}_{c}\right)\right] .
\end{aligned}
$$

Let $\mathcal{I}^{*}$ be the unknown image that produces a similar set of kriging error variances defined above: $L_{j}^{*}, j=1, \ldots, c$. The classification is carried out as follows:

$$
\text { Classify } \mathcal{I}^{*} \text { in } \omega_{s} \text { if } D_{\text {ave }}\left(\mathcal{I}^{*}, \omega_{s}\right)<D_{\text {ave }}\left(\mathcal{I}^{*}, \omega_{s^{\prime}}\right), \forall s^{\prime} \neq s,
$$

where $D_{\text {ave }}\left(\mathcal{I}^{*}, \omega_{s}\right)$ is the average difference of the variance errors of unknown image $I^{*}$ and image of class $\omega_{s}$. 


\section{$3 \quad$ Results}

The proposed method was tested to detect regions contaning mitochondria in microscope images of the intracellular space. The cells were imaged using scanning electron microscopy (SEM) and focused ion beam (FIB) technology. The dataset consists of 286 scans of the intracellular space. Figure 1 shows a typical FIB-SEM scan of a cancer cell line that was derived from a human head and neck squamous cell carcinoma (SCC-61) parental line [14, in which ground-truth mitochondria were identified and marked by a cell biologist. Figute 2 is a zoomed-in part of the SCC-61 intracellular space, showing mitochondria of various shapes across the intracellular structure.

The detection of the mitochondria in the intracellular space was carried out with a non-overlapping window of 53 by 60 pixels that is the average size of the mitochondria in the images. The window was moved along horizontal and vertical directions of the image to extract different features for training. For the FCM, $c=2$ and $\alpha=0.5,0.6,0.7,0.8$ and 0.9 . For the indicator kriging, $m=24(5 \times 5$ window $)$. To show the effectiveness of the feature extraction methods, only one image was used for training. For the purpose of comparisons with other methods, the training was performed by extracting the mean values of the gray-level co-occurrence matrix (GLCM), fractal dimension (FD), semi-variogram (SV), semi-variogram exponent (SVE), and the indicator-kriging co-occurrence matrix (IKCM) for mitochondrial and non-mitochondrial classes; which were studied in [15. If the image window contained whole or part of a mitochondrion, it was labelled as a mitochondrial object. This was designed to capture all small regions of interest containing mitochondria in order to maximize the sensitivity (true positive rate), while the specificity (true negative rate) can be first reasonably obtained and then maximized in the localized image segmentation task performed window by window. To validate the effectiveness of

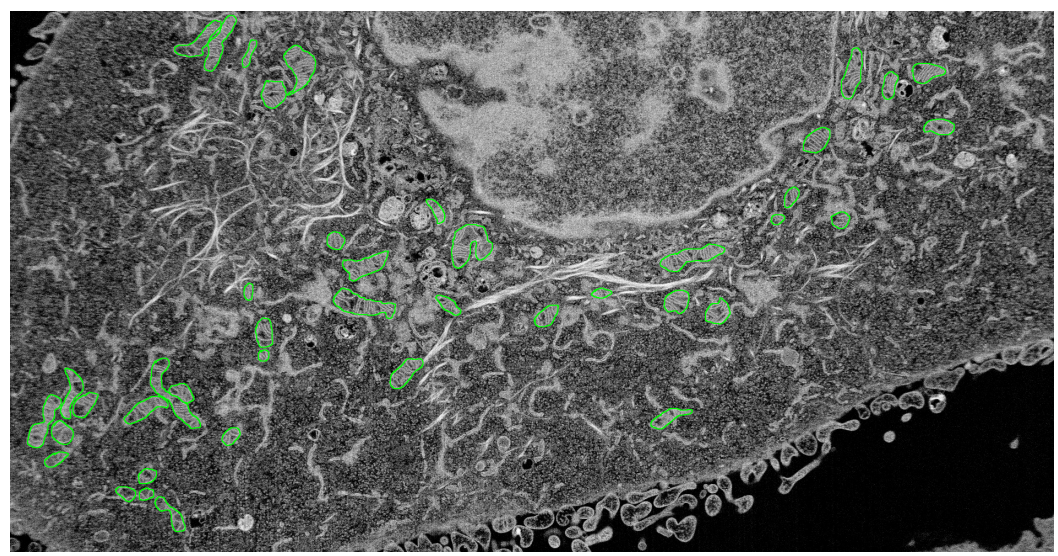

Fig. 1. An FIB-SEM image of SCC-61 in which the edges of ground-truth mitochondria were marked by a cell biologist 


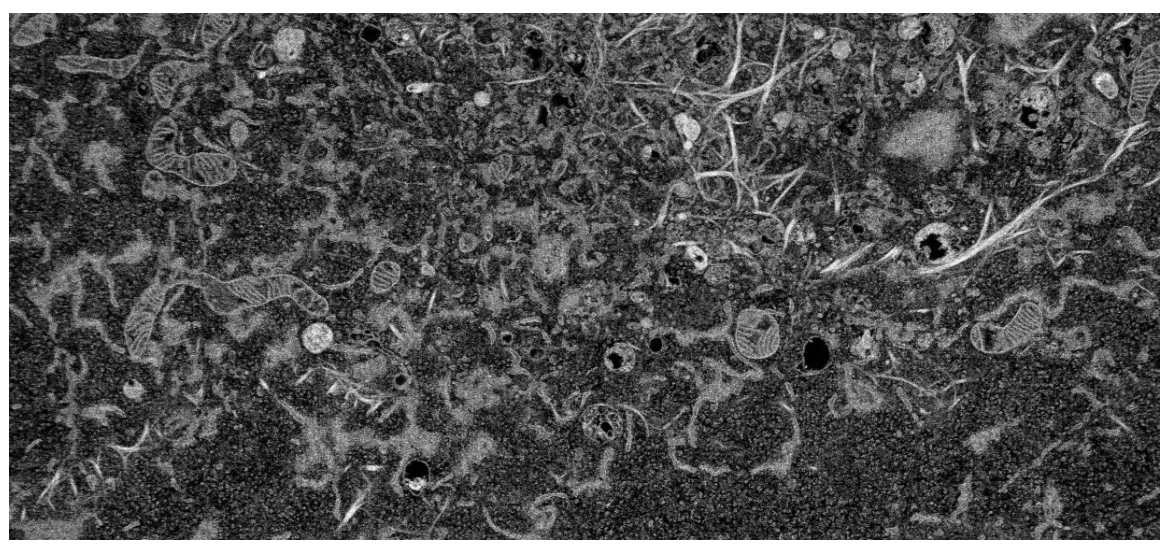

Fig. 2. A zoomed-in part of FIB-SEM image of SCC-61, in which mitochondria can be seen, having deep grooves and taking many shapes

Table 1. Sensitivity and Specificity using Different Methods

\begin{tabular}{lcccccc}
\hline \multicolumn{7}{c}{ GLCM FD SV } \\
\hline Sensitivity & 100 & 35 & 95 & 100 & 100 & 100 \\
Specificity & 5 & 90 & 70 & 50 & 87 & 94 \\
\hline
\end{tabular}

the extracted features, the Euclidean distance was used to calulate the similarity between the unknown sample and trained prototypes of the mitochondrial and non-mitochondrial objects.

Tables 1 shows the average sensitivity (SEN) and specificity (SPEC) of the experiments obtained from the proposed indicator kriging error difference method method (IKED) and other feature extraction methods using the Euclidean distance. The proposed IKED achieves the best results for both SEN and SPEC, while the IKCM yields the second best. The other methods in the decreasing order of classification performance for SEN are: GLCM = SVE, SV and FD; and for SPEC: FD, SV, SVE, and GLCM.

\section{Conclusion}

Methods for automated detection of mitochondria in complex miroscopy images of the real intracellular space of a cancer cell line have been presented and discussed. The experimental results demonstrated the effectiveness of the proposed method. The results can be combined to alleviate the task of image segmentation to tremendously reduce manual effort and speed up downstream biological analysis.

Acknowledgment. The image data were provided by Kazuhisa Ichikawa, Institute of Medical Science, the University of Tokyo. 


\section{References}

1. Gonzalez, R.C., Woods, R.E.: Digital Image Processing, 2nd edn. Prentice Hall, New Jersey (2002)

2. Iannaccone, P.M., Khokha, M.: Fractal Geometry in Biological Systems: An Analytical Approach. CRC Press, Boca Raton (1995)

3. Castellano, G., Bonilha, L., Li, L.M., Cendes, F.: Texture analysis of medical images. Clinical Radiology 59, 1061-1069 (2004)

4. Plissiti, M.E., Nikou, C., Charchanti, A.: Combining shape, texture and intensity features for cell nuclei extraction in Pap smear images. Pattern Recognition Letters 32, 838-853 (2011)

5. Zhang, B., Pham, T.D.: Phenotype recognition with combined features and random subspace classifier ensemble. BMC Bioinformatics 12, 128 (2011)

6. Nixon, M., Aguado, A.: Feature Extraction \& Image Processing, 2nd edn. Academic Press, Amsterdam (2008)

7. Zhou, Y., Huang, Y., Ling, H., Peng, J.: Medical image retrieval based on texture and shape feature co-occurrence. In: Proc. SPIE Medical Imaging 2012, vol. 8315, pp. 83151Q-83151Q-10 (2012)

8. Deutsch, C.V.: Geostatistical Reservoir Modeling. Oxford University Press, New York (2002)

9. Bezdek, J.C.: Pattern Recognition with Fuzzy Objective Function Algorithms. Plenum Press, New York (1981)

10. Isaaks, E.H., Srivastava, R.M.: An Introduction to Applied Geostatistics. Oxford University Press, New York (1989)

11. Davis, J.C.: Statistics and Data Analysis in Geology, 3rd edn. Wiley, New York (2002)

12. Leuangthong, O., Khan, K.D., Deutsch, C.V.: Solved Problems in Geostatistics. Wiley, New Jersey (2008)

13. Chiles, J.P., Delfiner, P.: Geostatistics: Modeling Spatial Uncertainty, 2nd ed. Wiley, New Jersey (2012)

14. Clark, E.S., Whigham, A.S., Yarbrough, W.G., Weaver, A.M.: Cortactin is an essential regulator of matrix metalloproteinase secretion and extracellular matrix degradation in invadopodia. Cancer Res. 67, 4227-4235 (2007)

15. Pham, T.D.: Automated identification of mitochondrial regions in complex intracellular space by texture analysis. In: Proc. SPIE, ICGIP 2013, 90690G, January 10, vol. 9069 (2014), doi:10.1117/12.2050102 